\title{
Influencia del entrenamiento pliométrico en la agilidad, una aproximación teórica
}

\section{Influence of plyometric training on agility, a theoretical approximation}

\author{
Wylmer Fernando Prieto-Barriga ${ }^{1}$ (iD
}

1Universidad Pedagógica y Tecnológica de Colombia,Tunja, Colombia; wylmer.prieto@uptc.edu.co

Cómo citar: Prieto-Barriga, W.F. 2021. Influencia del entrenamiento pliométrico en la agilidad, una aproximación teórica. Rev. Digit. Act. Fis. Deport. 7(2):e1615. http://doi.org/10.31910/rdafd.v7.n2.2021.1615

Artículo de acceso abierto publicado por Revista Digital: Actividad Física y Deporte, bajo una licencia Creative Commons CC BY-NC 4.0

Publicación oficial de la Universidad de Ciencias Aplicadas y Ambientales U.D.C.A, Institución de Educación Superior Acreditada de Alta Calidad por el Ministerio de Educación Nacional.

Recibido: marzo 19 de 2021 Aceptado: marzo 29 de 2021 Editado por: Álvaro José Gracia Díaz

\section{RESUMEN}

Introducción: La agilidad es, hoy, un factor clave en la formación de deportistas y su estudio en profundidad, diagnóstico y mejoramiento, a través de diversos métodos de entrenamiento, podría incidir, de manera directa, en el rendimiento de los deportistas de fútbol infantil. Objetivo: Realizar una aproximación teórica desde diferentes autores, que permita evidenciar la importancia de trabajar la agilidad dentro de la formación de futbolistas. Metodología: Se muestra el entrenamiento pliométrico como un método que puede favorecer el desarrollo de esta capacidad, a través de programas estructurados acordes a la formación de los niños. Se desarrolló una revisión bibliográfica, que tomó en cuenta artículos publicados desde el 2006 hasta el 2019, con el fin de encontrar estudios relacionados al desarrollo de la agilidad, por medio del entrenamiento pliométrico. La búsqueda fue basada en plataformas educativas, como Scielo, Scopus, DialNet, PubMed, ScienceDirect, Web of Science y Google scholar. Resultados: Después de esta búsqueda en las diferentes bases de datos, se encontraron, inicialmente, un total de 55 artículos relacionados con el objeto de estudio, de los cuales,
16 cumplieron con todos los criterios de inclusión. Conclusión: El entrenamiento pliométrico favorece el desarrollo de la agilidad, al mismo tiempo, que permite fortalecer algunas capacidades físicas en los niños futbolistas, entre los 11 y 13 años.

Palabras clave: Entrenamiento pliométrico; Agilidad; Fútbol.

\section{ABSTRACT}

Introduction: Agility is today a key factor in the training of athletes, and its in-depth study, diagnosis and improvement, through various training methods, could directly affect the performance of children's soccer athletes. Objective: make a theoretical approach from different authors, that shows the importance of working agility within the training of footballers. Methodology: Plyometric training is shown as a method that can promote the development of this ability, through structured programs according to the training of children. A bibliographic review was developed that took into account articles published from 2006 to 2019, in order to find studies related to the development of agility through plyometric 
training, the search was based on educational platforms such as Scielo, Scopus, DialNet, PubMed, ScienceDirect, Web Of science y Google scholar. Results: After this search in the different databases, a total of 55 articles related to the object of study were initially found, of which 16 met all the inclusion criteria. Conclusion: Plyometric training favors the development of complex coordinative abilities such as agility, at the same time that it allows strengthening some physical capacities in soccer players between 11 and 13 years old.

Keywords: Plyometric training; Agility; Soccer.

\section{INTRODUCCIÓN}

El fútbol es un deporte en conjunto de carácter intermitente, en el cual, gran parte de las acciones requieren un desarrollo significativo de la agilidad; de ahí, la importancia de trabajar esta capacidad coordinativa, como eje fundamental de cada sesión de entrenamiento, en niños entre los 11 y 13 años, de modo que estos deportistas, en formación, puedan realizar acciones más rápidas y explosivas, que potencien lo individual y se conviertan en parte importante dentro del desarrollo de un partido; en tal sentido, se hace necesario identificar los diferentes elementos que componen esta capacidad.

Resulta oportuno hacer una aproximación conceptual, con el ánimo de realizar una descripción teórica sobre la agilidad, que permita identificar los elementos que la constituyen y que podrían ser la base para abordarla dentro de la planificación del entrenamiento, brindándole la importancia que merece, en la formación deportiva.

En este sentido, los estudios han mostrado diferentes métodos de entrenamiento para el trabajo de la agilidad, con jugadores de distintas edades de formación, en busca de un mayor rendimiento deportivo. En la indagación previa, uno de esos métodos sobresalientes ha sido el entrenamiento pliométrico, que es planteado como herramienta para el desarrollo de la agilidad, en atletas practicantes, de diversas disciplinas deportivas.

Es importante visualizar la relevancia de este tipo de entrenamientos en la formación de deportistas, con edades entre los 11 y 13 años, además de sus efectos sobre esta capacidad, permitiendo que los entrenadores, de etapas formativas, tengan más herramientas, para potenciar sus deportistas y consolidar un desarrollo, acorde a la edad y a la etapa de formación.

A lo largo del tiempo, la noción de agilidad ha ido variando y evolucionando, debido a los diversos estudios que se han realizado, centrados en el desarrollo de esta capacidad. Una primera aproximación, al término actual, fue la dada por Draper \& Lancaster (1985), quienes conceptualizaban la agilidad como la velocidad necesaria para realizar cambios de dirección, un concepto que dejaba de lado muchos otros aspectos, que hacen parte de esta capacidad; además, no genera, en sí, importancia en su entrenamiento.

Posteriormente, el término fue evolucionando y agregando aspectos que serían importantes al momento de indagar el desarrollo de esta capacidad y su relevancia en el rendimiento deportivo.

La agilidad, se relaciona con dos tipos de funciones motoras. Por un lado, es crucial para la capacidad de arrancar de forma explosiva, desacelerar, cambiar de dirección y acelerar de nuevo, rápidamente, mientras se mantiene el control del cuerpo y se minimiza la pérdida de velocidad. Por otra parte, la agilidad también se refiere a la capacidad de coordinar varias tareas de deportes específicos simultáneamente (Costello \& Kreis, 1993), mostrando, que esta capacidad coordinativa compleja, está constituida por varios elementos encadenados, que son importantes y que se deben trabajar, para lograr el desarrollo óptimo, dentro de la práctica deportiva.

Por otro lado, Mori et al. (1995) afirman: "La agilidad es la capacidad de realizar una secuencia de movimientos globales a máxima velocidad, con cambios de dirección, y sobre los tres planos del espacio, generalmente, en situaciones imprevistas". Con esta definición, se puede evidenciar dos factores comunes e importantes, en estos tres primeros conceptos; el primero de ellos es la velocidad, que es una constante dentro de la conceptualización del término y que remarca su importancia para el desarrollo de esta capacidad; el segundo está centrado en el cambio de dirección que se plantea como el fin, al cual, debe llegar la agilidad; por su parte, estos autores agregan las situaciones imprevistas, como un factor clave en su noción, debido a que muchas de las 
acciones que ejecuta un deportista en la práctica son determinadas por el contexto y las situaciones que, en su momento, deben enfrentar, para lo cual, ésta capacidad debe ser la clave.

Asimismo, otras definiciones, como "La agilidad es un movimiento rápido de todo el cuerpo, con cambio de velocidad o dirección en respuesta a un estímulo" (Sheppard \& Young, 2006), dada tras el análisis devarios postulados, que permite una mejor contextualización de la agilidad dentro del entrenamiento, debido a que, estos autores, evidencian una relación estrecha entre esta capacidad, algunas cualidades físicas y ciertos elementos cognitivos, donde se podrían centrar puntos de partida, para el entrenamiento de la agilidad, no solo como parte global sino como un elemento central.

Ahora bien, acercándonos un poco más al contexto del fútbol, se encuentran aportes interesantes al concepto: "La capacidad de combinar fuerza muscular, fuerza de arranque, fuerza explosiva, balance, aceleración y desaceleración determinan la agilidad" (Rivas \& Sánchez, 2013). Esta mirada, se centra en algunos elementos que se deben trabajar, según los autores, para lograr el desarrollo de esta capacidad coordinativa compleja, teniendo en cuenta que, varios de ellos, se llevan a cabo en las sesiones de entrenamiento que desarrollan los equipos regularmente, poniendo a la agilidad, como un elemento resultante de otros trabajos y no como un elemento importante que se deba trabajar de manera individualizada.

De igual manera, Letona et al. (2015) afirma que "La agilidad se define como la capacidad de cambiar la dirección del cuerpo rápidamente y es el resultado de una combinación de fuerza, velocidad, equilibrio y coordinación". Un concepto que resalta la importancia del trabajo de capacidades físicas, combinadas con capacidades coordinativas especiales y que pone a la agilidad, como un elemento importante que merece un trabajo particular, dentro de la planificación del entrenamiento.

Con referencia a lo anterior, se puede cerrar este contexto, con la construcción de un concepto, abarcando aspectos importantes, relacionados en los postulados de los diferentes autores mencionados: La agilidad es el resultado de la combinación de fuerza, velocidad, equilibrio y coordinación, que le permiten al deportista cambiar de dirección rápidamente, sin perder el balance y el control del cuerpo, respondiendo a diferentes estímulos.

Avanzando en este razonamiento, es importante conceptualizar el término entrenamiento pliométrico, que es otra variable importante de esta revisión teórica; este es un concepto que comienza a tener auge hacia los años noventa, con el profesor Verkhoshansky (1999), quien afirma que "Es una forma específica de preparación de la fuerza dirigida al desarrollo de la fuerza explosiva muscular y de la capacidad reactiva del sistema neuromuscular. Este método es un medio de preparación física especial". Esta noción muestra el camino que, durante años, este tipo de trabajo tuvo dentro de la preparación física de los deportistas, por sus resultados y su facilidad, en cuanto a la aplicación, tomó auge rápidamente y fue una constante en las investigaciones, que buscaban obtener resultados específicos, de la manera más oportuna y rápida.

De igual manera, el concepto fue evolucionando, a través del tiempo, con los avances que se iban dando en los diferentes estudios. Los ejercicios pliométricos son definidos como aquellos que capacitan a un músculo, a alcanzar una fuerza máxima en un periodo de tiempo, lo más corto posible (Chu, 2006). En esta conceptualización, el autor se centra en el resultado que debe traer la aplicación de los ejercicios pliométricos, lo que permite visibilizar un poco más, hacia donde debe ir este tipo de trabajos y la importancia que deben tener en función del tiempo, debido a que su implementación debe permitir resultados en un menor tiempo y, así, marcar una diferencia con cualquier otro método de entrenamiento.

Por último, es importante, dentro de este concepto, destacar el aporte que hace Cappa (2000), quien afirma:

Se puede definir a la pliometría, como un método de entrenamiento de la fuerza explosiva, que utiliza la acumulación de energía en los componentes elásticos del músculo y los reflejos durante la fase excéntrica de un movimiento, para su posterior utilización y potenciación, durante la fase concéntrica.

Esta noción permite ver una descripción del término más desde lo fisiológico, nombrando los componentes musculares y las fases que se desarrollan con el trabajo, dejando, en evidencia, la importancia de realizar ejercicios adecuados, que logren la activación 
de estos componentes y que, en el trascurrir del entrenamiento, los resultados puedan ser más visibles y duraderos, a través de estímulos adecuados.

\section{MATERIALES Y MÉTODOS}

Se desarrolló una revisión bibliográfica, que tomó en cuenta artículos publicados desde el 2006 hasta el 2019, con el fin de encontrar estudios relacionados al desarrollo de la agilidad, a través del entrenamiento pliométrico; la búsqueda fue basada en plataformas educativas, como Scielo, Scopus, DialNet, PubMed, ScienceDirect, Web Of science y Google scholar, a través de palabras claves, como entrenamiento pliométrico, agilidad y fútbol.

De igual manera, se elaboró un formato para la identificación de los elementos importantes de cada uno de los documentos, libros y artículos, que fueron encontrados, a través del rastreo bibliográfico, donde se resaltaron datos, como título, autor, tipo de documento, variables, año, país, editorial y base de datos.

Criterios de inclusión y exclusión. Se identificaron un total de 55 artículos, para la selección de los estudios más relevantes, se realizó una lectura analítica y se diligenció el formato establecido, teniendo en cuenta 3 criterios importantes: como primer criterio, el estudio no debía exceder las fechas establecidas ( $n=55)$; el segundo fue la relación de los estudios con dos de las tres palabras claves, como mínimo $(n=26)$ y último criterio, se estableció el entrenamiento pliométrico, como tema relevante dentro de cada estudio $(n=16)$.

En el proceso de exclusión se tuvieron en cuenta varios ítems, como se observa en la figura 1.

Tabla 1. Resumen de estudios encontrados y sus hallazgos.

\begin{tabular}{|c|c|c|c|c|}
\hline Autor, año & Edad & Test & $\begin{array}{l}\text { Tiempo de } \\
\text { aplicación }\end{array}$ & Resultados \\
\hline $\begin{array}{l}\text { Miller et al. } \\
(2006)\end{array}$ & $\begin{array}{l}\text { GC } 24,2 \pm \\
4,8 ; \text { GE } 22,3 \\
\pm 3,1 \text { años }\end{array}$ & $\begin{array}{l}\text { Prueba } T \text {, test de Illinois, placa de } \\
\text { fuerza }\end{array}$ & $\begin{array}{l}6 \text { semanas, } \\
\text { dos veces por } \\
\text { semana }\end{array}$ & $\begin{array}{l}\text { Para la prueba } T \text {, los tiempos mejoraron en un } 4,86 \% \text {, para la } \\
\text { prueba de agilidad de Illinois, } 2,93 \% \text { y para la placa de fuerza, los } \\
\text { sujetos mejoraron en más del } 10 \%\end{array}$ \\
\hline $\begin{array}{l}\text { Meylan \& } \\
\text { Malatesta } \\
(2009)\end{array}$ & $\begin{array}{c}13,36 \pm 0,6 \\
\text { años }\end{array}$ & $\begin{array}{l}\text { Salto en cuclillas (SJ), salto con } \\
\text { contramovimiento (CMJ) y prueba } \\
\text { de contacto }(\mathrm{CT}) \text {, prueba múltiple de } \\
5 \text { saltos, sprint de } 10 \mathrm{~m} \text {, cuatro } \\
\text { cambios de dirección de } 60 \text { grados } \\
\text { en } 10 \mathrm{~m}\end{array}$ & $\begin{array}{l}8 \text { semanas, } \\
\text { dos veces por } \\
\text { semana. }\end{array}$ & $\begin{array}{l}\text { Aumento significativo en la altura de salto en } \mathrm{CMJ}(+7,9 \%) \text { y CT } \\
(+10,9 \%) \text {, pero no SJ }(+0,6 \%) \text {; disminución significativa } \\
\text { en el tiempo de sprint de } 10 \mathrm{~m}(22,1 \%) \text {; disminución } \\
\text { significativa del } 9,6 \%(4,69 \pm 0,16 \text { a } 4,24 \pm 0,17 \text { segundos; } p \text {, } \\
\text { 0,001) en el tiempo de prueba de agilidad }\end{array}$ \\
\hline $\begin{array}{l}\text { Thomas et } \\
\text { al. (2009) }\end{array}$ & $\begin{array}{l}17,3 \pm 0,4 \\
\quad \text { años }\end{array}$ & $\begin{array}{l}\text { Salto con contramovimiento (CMJ); } \\
\text { Velocidad en } 20 \text { metros; prueba de } \\
\text { agilidad } 505\end{array}$ & $\begin{array}{l}6 \text { semanas, } \\
\text { dos veces por } \\
\text { semana }\end{array}$ & $\begin{array}{l}\text { Hubo aumentos en la altura del salto vertical }(F[1,10]= \\
42,22, p<0,05 \text {, y disminuciones en los tiempos de agilidad }(F[1 \text {, } \\
10]=60,97, p<0,05 \text {, para ambos grupos. No hubo cambios } \\
\text { en la velocidad de sprint }(F[1,10]=0,14, p>0,05\end{array}$ \\
\hline $\begin{array}{l}\text { Roopchand } \\
\text { \& Lue } \\
(2010)\end{array}$ & $>18$ años & $\begin{array}{l}\text { Illinois, placa de fuerza, salto } \\
\text { vertical, salto largo sin impulso }\end{array}$ & $\begin{array}{l}3 \text { semanas; } \\
\text { dos veces por } \\
\text { semana }\end{array}$ & $\begin{array}{l}\text { Las puntuaciones medias de salto vertical al final de las tres } \\
\text { semanas fueron de }(1,8 \pm 2,7 \mathrm{~cm}, p=0,023) \text {, las } \\
\text { puntuaciones de salto amplio de }(12,04 \pm 12,11 \mathrm{~cm}, \mathrm{p}= \\
\text { 0002), y se evidenció un cambio medio en las puntuaciones de } \\
\text { agilidad }(0,45 \pm 0,79 \mathrm{seg}, p=0,045)\end{array}$ \\
\hline $\begin{array}{l}\text { Ceballos \& } \\
\text { Zaraza } \\
(2012)\end{array}$ & 18 años & Salto de Longitud, salto vertical & $\begin{array}{l}1-2 \text { sesiones } \\
\text { por semana }\end{array}$ & $\begin{array}{l}\text { El trabajo pliométrico durante la preparación física de los } \\
\text { futbolistas juveniles es de vital importancia, para desarrollar la } \\
\text { fuerza explosiva en este deporte. A través de los ejercicios } \\
\text { pliométricos propuestos es posible incrementar la fuerza } \\
\text { explosiva en el tren inferior de los futbolistas; estos son flexibles } \\
\text { en dependencia de las características del equipo }\end{array}$ \\
\hline
\end{tabular}




\section{Continuación tabla 1.}

\begin{tabular}{|c|c|c|c|c|}
\hline Autor, año & Edad & Test & $\begin{array}{l}\text { Tiempo de } \\
\text { aplicación }\end{array}$ & Resultados \\
\hline $\begin{array}{l}\text { Michailidis } \\
\text { et al. (2013) }\end{array}$ & $\begin{array}{l}\mathrm{GC}, \mathrm{n}=21 \\
10,6 \pm 0,5 \\
\quad \text { años, } \\
\text { GPT } \mathrm{n}=24 \\
10,6 \pm 0,6 \\
\quad \text { años }\end{array}$ & $\begin{array}{l}\text { Prueba de sprint de } 30 \mathrm{~m} \text {, con } \\
\text { divisiones de } 10 \mathrm{~m} \text {, salto de } \\
\text { longitud, prueba múltiple de } 5 \\
\text { límites, prueba de salto vertical, } \\
\text { prueba de sentadilla con barra de } \\
\text { 10RM, prueba de Wingate de } 30 \\
\text { segundos }\end{array}$ & $\begin{array}{l}12 \text { semanas, } \\
\text { tres veces por } \\
\text { semana }\end{array}$ & $\begin{array}{l}\text { Ambos grupos demostraron una marcada reducción de los } \\
\text { tiempos en el sprint. El GPT mejoró los } 3 \text { tipos de saltos al } \\
\text { final de la intervención }(23,3 \% \text { en SJ, } 27,6 \% \text { en CMJ y } \\
15,9 \% \text { en DJ, } p<0,05) \text {. En el rendimiento del salto de } \\
\text { longitud GPT obtuvo un } 4,2 \% \text {, } p<0,05 \text {. En el rendimiento de } \\
\text { la agilidad mejoró el GPT, en un } 22,8 \%, p<0,05 \text { y la } \\
\text { distancia de patada mejoró un } 22,5 \%, p<0,05\end{array}$ \\
\hline $\begin{array}{l}\text { Söhnlein et } \\
\text { al. (2014) }\end{array}$ & $\begin{array}{l}11,2 \pm 14,7 \\
\text { años }\end{array}$ & $\begin{array}{l}\text { Pruebas de sprint de } 30 \mathrm{~m} \text { y } 20 \mathrm{~m} \text {, } \\
\text { The hurdle agility run (HAR), AX5 } \\
10-\mathrm{m} \text { Shuttle Run, Multiple } 5 \\
\text { Bounds, Salto de longitud de pie }\end{array}$ & $\begin{array}{l}16 \text { semanas, } \\
\text { dos veces por } \\
\text { semana }\end{array}$ & $\begin{array}{l}\text { El grupo de entrenamiento pliométrico reveló mejoras en el } \\
\text { rendimiento en todas las pruebas, con la excepción del } \\
\text { sprint de } 10 \mathrm{~m} \text { y el rendimiento SR de } 5 \times 10 \mathrm{~m} \text {. Todos los } \\
\text { efectos significativos se clasifican como grandes }\end{array}$ \\
\hline $\begin{array}{l}\text { Pérez et al. } \\
(2015)\end{array}$ & Todas & $\mathrm{N} / \mathrm{A}$ & N/A & $\begin{array}{l}\text { La revisión llevada a cabo nos demuestra que la agilidad de } \\
\text { los futbolistas puede ser mejorada desde un } 0,8 \% \text { hasta un } \\
22,8 \% \text {, en función del test utilizado: No existe ningún test } \\
\text { de agilidad universalmente utilizado por todos para medir } \\
\text { esta cualidad; de hecho, entre los } 20 \text { artículos incluidos en } \\
\text { la revisión, hay } 17 \text { test de agilidad utilizados }\end{array}$ \\
\hline $\begin{array}{l}\text { Bedoya et } \\
\text { al. (2015) }\end{array}$ & 10 a 17 años & $\mathrm{N} / \mathrm{A}$ & N/A & $\begin{array}{l}\text { El entrenamiento pliométrico, se debe completar } 2 \text { días por } \\
\text { semana, durante } 8-10 \text { semanas, con un período de } \\
\text { descanso de } 72 \text { horas, entre los días de entrenamiento } \\
\text { pliométrico. El tiempo total para el entrenamiento } \\
\text { pliométrico debe durar entre } 10 \text { y } 25 \text { minutos. El número } \\
\text { inicial de contactos de pie debe ser de } 50 \text { a } 60 \text { por sesión y } \\
\text { aumentar a no más de } 80 \text { a } 120 \text { contactos de pie por sesión. } \\
\text { Se deben realizar de tres a cuatro ejercicios de } \\
\text { entrenamiento pliométrico de } 2 \text { a } 4 \text { series de } 6 \text { a } 15 \\
\text { repeticiones por sesión de entrenamiento }\end{array}$ \\
\hline $\begin{array}{l}\text { Celis \& } \\
\text { Sabogal } \\
\text { (2016) }\end{array}$ & 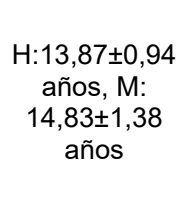 & $\begin{array}{l}\text { Salto horizontal sin impulso, las } \\
\text { pruebas de velocidad de } 20 \\
\text { metros lanzados y de agilidad de } \\
10 \times 5 \text { metros. }\end{array}$ & $\begin{array}{l}8 \text { semanas, } \\
\text { tres veces a } \\
\text { la semana }\end{array}$ & $\begin{array}{l}\text { En el salto horizontal los hombres aumentaron } 17,8 \mathrm{~cm} \text {. Las } \\
\text { mejoras en el test de } 20 \text { metros fueron de } 0,36 \mathrm{~s} \text {, en los } \\
\text { hombres y } 0,19 \mathrm{~s} \text {, en las mujeres. En cuanto a agilidad } \\
\text { existieron mejoras, tanto en hombres como en mujeres, } \\
\text { disminuyendo el tiempo inicial, en } 0,81 \mathrm{~s} \text { y } 0,28 \mathrm{~s} \text {, } \\
\text { respectivamente }\end{array}$ \\
\hline $\begin{array}{l}\text { Moreno } \\
(2017)\end{array}$ & $\begin{array}{l}14,73 \pm 0,59 \\
\text { años }\end{array}$ & $\begin{array}{l}\text { Test de sprint lineal } 20 \mathrm{~m} \text {, Test de } \\
\text { agilidad: consistía en cuatro } \\
\text { cambios de dirección de } 60 \\
\text { grados sobre } 10 \mathrm{~m} \text {, Test de salto } \\
\text { vertical. }\end{array}$ & $\begin{array}{l}6 \text { semanas, } \\
\text { dos días por } \\
\text { semana }\end{array}$ & $\begin{array}{l}\text { Se encontraron diferencias significativas }(p \leq 0,05) \text {, en el test } \\
\text { de sprint de } 20 \mathrm{~m} \text { lineales en el GE }(-0,19 \text { segundos, } 5,5 \% \text {, } \\
\text { TE }=1,1) \text {; Salto vertical }(p \leq 0,05) \mathrm{GE}(2,9 \mathrm{~cm}, 7,1 \% \text {, TE }=0,3) \\
\text { y test de agilidad, diferencias significativas con }(p \leq 0,05) \text { en } \\
\text { el GE, empezando por el lado izquierdo }[\mathrm{GE}(-0,15 \\
\text { segundos, } 2,7 \% \text {, TE }=0,6)] \text { y empezando por el lado } \\
\text { derecho }[\mathrm{GE}(-0,16 \text { segundos, } 2,8 \% \text {, TE }=0,5)]\end{array}$ \\
\hline $\begin{array}{l}\text { Makhlouf et } \\
\text { al. (2018) }\end{array}$ & 10 a 12 años & $\begin{array}{l}\text { Salto con contramovimiento CMJ, } \\
\text { Prueba de triple salto THT, } 4 \mathrm{~m} \times \\
9 \text {-m shuttle run, Illinois change of } \\
\text { direction test (ICODT)) con y sin } \\
\text { balon, sprints de } 10 \text { a } 30 \mathrm{~m} \text {, } \\
\text { Equilibrio en } \mathrm{Y}\end{array}$ & $\begin{array}{l}8 \text { semanas, } \\
\text { dos veces por } \\
\text { semana }\end{array}$ & $\begin{array}{l}\text { Todas las medidas mostraron mejoras significativas en el } \\
\text { efecto principal. Los efectos principales significativos para } \\
\text { el grupo fueron evidentes con el ICODT con y sin balón } \\
\text { (Trivial), THT (Trivial), prueba de equilibrio del trabajo de } \\
\text { pie (Pequeño) y efecto casi significativo ( } p=0,058 \text {; trivial) } \\
\text { con el CMJ. Lo que evidencia mejoras significativas y } \\
\text { similares de BPT, APT, después de } 8 \text { semanas de } \\
\text { entrenamiento versus control para esprintar, cambio de } \\
\text { dirección (ICODT), agilidad, así como la prueba de } \\
\text { equilibrio en Y }\end{array}$ \\
\hline $\begin{array}{l}\text { Fischetti et } \\
\text { al. (2019) }\end{array}$ & $\begin{array}{l}\text { GC } 26,7 \pm 5,3 \\
\text { años, GP } \\
26,5 \pm 6,9 \\
\text { años }\end{array}$ & $\begin{array}{l}\mathrm{T} \text { test, Salto } \\
\text { contramoviemiento CMJ }\end{array}$ & $\begin{array}{l}12 \text { semanas, } \\
\text { tres veces por } \\
\text { semana }\end{array}$ & $\begin{array}{l}\text { El análisis post-hoc en CMJ reveló un aumento significativo } \\
\text { en la altura máxima alcanzada antes y después de la } \\
\text { prueba en el PG }(p=0,0003, d=0,55) \text {; de igual manera, el } \\
\text { análisis del T-test post hoc reveló una disminución } \\
\text { significativa en el tiempo de ejecución en el PG }(p=0,0005 \text {, } \\
d=0,73)\end{array}$ \\
\hline
\end{tabular}


Continuación tabla 1.

\begin{tabular}{|c|c|c|c|c|}
\hline Autor, año & Edad & Test & $\begin{array}{l}\text { Tiempo de } \\
\text { aplicación }\end{array}$ & Resultados \\
\hline $\begin{array}{l}\text { Tottori et al. } \\
\text { (2019) }\end{array}$ & 9 a 12 años & $\begin{array}{l}\text { Sprint de } 50 \mathrm{~m} \text {, salto de } \\
\text { contramovimiento (CMJ), salto en } \\
\text { cunclillas (SJ) y saltos de rebote (RJ) }\end{array}$ & $\begin{array}{l}8 \text { semanas, } \\
\text { una vez por } \\
\text { semana }\end{array}$ & $\begin{array}{l}\text { El grupo de entrenamiento polimétrico mostró una } \\
\text { velocidad de impresión mejorada a } 20-30 \mathrm{~m}, 30-40 \text { y } 40-50 \mathrm{~m} \\
\text { y longitud de paso a } 0-10 \mathrm{~m}, 20-30 \mathrm{~m} \text { y } 30-40 \mathrm{~m}(\mathrm{p}<0,05) \text {. } \\
\text { Además, solo el grupo pliométrico mostró una mayor } \\
\text { distancia en el salto de longitud y rendimiento en el salto de } \\
\text { rebote }(p<0,05) \text {; esto sugiere, que el entrenamiento pliométrico } \\
\text { en niños preadolescentes mejora la velocidad del sprint y la } \\
\text { longitud del paso en la fase de velocidad máxima, } \\
\text { concomitante con un mayor rendimiento en el salto } \\
\text { horizontal y de rebote }\end{array}$ \\
\hline $\begin{array}{l}\text { Branquinho } \\
\text { et al. (2019) }\end{array}$ & $\begin{array}{c}17,27 \pm 0,458 \\
\text { años }\end{array}$ & $\begin{array}{l}\text { Salto con contramovimiento, } \\
\text { regate de velocidad, sprint de } 30 \\
\text { metros, velocidad de patadas y } \\
\text { una prueba de recuperación } \\
\text { intermitente de recuperación Yo-Yo } \\
\text { Nivel } 2 \text { (Yo-Yo IE2) }\end{array}$ & $\begin{array}{l}8 \text { semanas, } \\
\text { dos veces por } \\
\text { semana }\end{array}$ & $\begin{array}{l}\text { Los resultados mostraron diferencias estadísticamente } \\
\text { significativas }(p<0,05) \text {, en el rendimiento del sprint, salto con } \\
\text { contramovimiento, Yo-yo IE } 2 \text { y regate de velocidad. La velocidad } \\
\text { máxima con él no presentó diferencias } \\
\text { estadísticamente significativas }(p>0,05) \text {. Estos resultados } \\
\text { demuestran que la combinación de ejercicios de fútbol y } \\
\text { entrenamiento de potencia específico, sin tiempo de } \\
\text { entrenamiento adicional, durante la temporada, optimiza un } \\
\text { rendimiento general y específico del fútbol }\end{array}$ \\
\hline $\begin{array}{l}\text { Şeker et al. } \\
\text { (2019) }\end{array}$ & $21,5 \pm 2$ años & $\begin{array}{l}\text { VO2máx, fuerza de piernas, salto } \\
\text { vertical, Wingate anaeróbico, salto } \\
\text { de longitud de pie y velocidad en } 30 \\
\text { metros }\end{array}$ & $\begin{array}{l}8 \text { semanas, } \\
\text { tres veces a } \\
\text { la semana }\end{array}$ & $\begin{array}{l}\text { Hubo una diferencia estadísticamente significativa en los } \\
\text { valores pretest y postest del grupo experimental, en } \\
\text { términos de salto vertical, salto amplio de pie, velocidad de } 30 \\
\text { metros, fuerza de piernas y parámetros de potencia } \\
\text { anaeróbica del entrenamiento pliométrico }(p<0,05) \text {, lo cual, } \\
\text { determinó que el entrenamiento pliométrico de } 8 \text { semanas } \\
\text { tuvo efectos positivos en los valores de rendimiento de los } \\
\text { sujetos }\end{array}$ \\
\hline
\end{tabular}

$\mathrm{GE}=$ grupo experimental, GC = grupo control, GP/GPT/PG = grupo pliométrico, $\mathrm{CJ}=$ salto con contramovimiento, SLJ = salto de longitud de pie,

$\mathrm{SJ}=$ salto en cuclillas, $\mathrm{DJ}=$ salto con caída, LS = paso lateral, ICODT = Prueba de cambio de dirección de Illinois, THT= Prueba de triple salto,

$\mathrm{CT}=$ prueba de contacto.

\section{RESULTADOS Y DISCUSIÓN}

Después de hacer la búsqueda en las diferentes bases de datos, a través de palabras claves, como agilidad, entrenamiento pliométrico y fútbol, se encontraron, inicialmente, un total de 55 artículos, relacionados con estos términos, de los cuales, 16 cumplieron con todos los criterios de inclusión. Estos estudios tuvieron una lectura rigurosa, para extraer la información más relevante, en cuanto al objeto de estudio.

Para empezar, es importante resaltar que la evaluación de la agilidad se ha convertido en parte esencial del proceso de entrenamiento, aunque es evidente, que cada disciplina deportiva le ha dado un enfoque distinto, con el ánimo de obtener resultados más parecidos, a la especificidad de la competencia.

En segundo lugar, hay que mencionar que, aproximadamente, desde los años ochenta, en Estados Unidos, se empezaron a ver las posibles aplicaciones del método de entrenamiento pliométrico en deportes de conjunto, con el fin de mejorar los programas deportivos; en este camino, se encontraron con muchos inconvenientes, por la falta de experiencia de los entrenadores en el manejo del entrenamiento pliométrico o por la capacidad de los deportistas.

Entrenamiento pliométrico para el desarrollo de la agilidad. Con el tiempo, los entrenadores introdujeron, paulatinamente, a sus programas de entrenamiento, algunos ejercicios pliométricos, con el propósito de mejorar el rendimiento de sus deportistas; muchos de ellos, lo consideraron como un método de baja complejidad en su aplicación, debido a que no requerían demasiados implementos adicionales para ponerlo en práctica, dentro de una sesión de entrenamiento, de cualquier nivel deportivo.

Es por esto, que se pueden evidenciar estudios, donde el entrenamiento pliométrico es propuesto como uno de los métodos que influye en el desarrollo de las capacidades físicas y coordinativas de los deportistas, debido a que ha sido comparado, contrastado, en otras oportunidades, trabajado de manera simultánea o como complemento de otros métodos, con el fin de evidenciar, de qué manera se puede generar mejor desarrollo, de acuerdo con el objeto de estudio. Además, Ceballos \& Zaraza (2012) afirmaron que "El ejercicio pliométrico es uno de los métodos de entrenamiento más disponibles y eficientes con el tiempo, y podría decirse, que brinda la mayor 
posibilidad de transferencia para su aplicación en el deporte", lo que facilitaría, aún más, su inmersión dentro del entrenamiento regular de un equipo o un atleta en preparación.

En tal sentido, es importante mencionar el estudio elaborado por Miller etal. (2006), donde identificaron, mediante EI ANCOVA univariante, un efecto grupal significativo, con $p=0,0000$, con una mejora del $4,86 \%$, para la prueba $T$ de agilidad; para la prueba de agilidad de Illinois, también encontraron un efecto de grupo significativo con $p=0,000$, con una mejora del $2,93 \%$ y, finalmente, para la prueba Force Plate $p=0,002$, con una mejora del $10 \%$, después de una intervención de 6 semanas de entrenamiento pliométrico, con jóvenes atletas. Estos resultados evidenciaron que el entrenamiento pliométrico puede ser una técnica de entrenamiento efectiva, para mejorar la agilidad.

Por otro lado, Roopchand \& Lue (2010) encontraron mejoras de 0,45 $\pm 0,79$ segundos en promedio, después de la aplicación de un entrenamiento pliométrico de tres semanas, datos estadísticamente significativos con $p=0,045$, con lo cual, se puede concluir, que un entrenamiento pliométrico de corta duración puede llevar a mejoras significativas en el rendimiento y la agilidad, en jugadoras de Netball.

Para terminar esta parte, se resalta la revisión sistemática sobre el entrenamiento de agilidad en futbolistas, adelantada por Pérez et al. (2015), quienes dieron una mirada al estado de la investigación sobre el entrenamiento de agilidad y cómo se podía mejorar dicha capacidad; su búsqueda, se basó en dos palabras claves: agility y training soccer, dando como resultado un total de 99 publicaciones, de las cuales, solo 20 cumplieron como artículos funcionales, con los criterios de la revisión.

De este estudio, se pudo concluir, que la agilidad se puede mejorar durante la pretemporada, la temporada o una vez terminada la temporada; estas mejoras, se pueden dar bajo métodos de entrenamiento con pesas, entrenamiento combinado de fuerza y resistencia, entrenamiento de contraste, juegos con balón en espacios reducidos, entrenamientos de Sprint con cambios de dirección, entrenamientos neuromusculares y entrenamiento pliométrico, que pueden ser desarrollados con futbolistas amateur o profesionales y su mejora oscila desde un 0,8 hasta un $22,8 \%$.
Todo lo anterior permite evidenciar cómo se ha ido integrando el entrenamiento pliométrico a la planificación de los deportes en conjunto, dejando claro que puede no solo tener efectos positivos en la agilidad, sino en otras capacidades físicas, permitiendo, así, un desarrollo integral de los deportistas, que utilizan este tipo de trabajos.

Entrenamiento pliométrico en deportistas jóvenes. Algunos supuestos proponían que el entrenamiento pliométrico era perjudicial para la población juvenil, debido a que aumentaba el riesgo de lesiones y generaba un retraso en el crecimiento; sin embargo, con el transcurso del tiempo, se ha demostrado que es beneficioso para los atletas jóvenes, cuando se siguen las pautas apropiadas para la edad, como las recomendadas dadas por la Sociedad Canadiense de Fisiología del Ejercicio (CSEP) (Bedoya et al. 2015). Si bien es un método exigente físicamente, no está tan lejos de las acciones que realizan los niños y los jóvenes en otras actividades recreativas o deportivas, en que se encuentra inmersos, habitualmente.

Por esto, es importante empezar a referenciar estudios, como el realizado por Meylan \& Malatesta (2009), con el cual, buscaron evidenciar el efecto del entrenamiento pliométrico de 8 semanas, dentro de la práctica de fútbol en temporada, sobre acciones explosivas en jugadores jóvenes. Los resultados de este estudio asociaron el entrenamiento pliométrico con disminuciones significativas en el tiempo de carrera de $10 \mathrm{~m}$ $(-2,1 \%)$ y tiempo de prueba de agilidad $(-9,6 \%)$. Además, mostraron aumentos significativos en la altura de salto para CMJ $(+7,9 \%)$ y CT $(+10,9 \%)$. Estos datos evidencian los beneficios que tiene un programa de entrenamiento pliométrico en las acciones explosivas de los jugadores jóvenes.

De la misma manera, Tottori et al. (2019) realizaron un trabajo denominado Efectos del entrenamiento pliométrico sobre el rendimiento en carreras de sprint en niños de 9 a 12 años, donde también utilizaron un programa de entrenamiento de 8 semanas. Como resultado de este trabajo, se pudo evidenciar que el grupo de entrenamiento pliométrico mostró mejoras en la velocidad de sprint y la longitud de paso $(p<0,05)$; además, mostró un aumento en la distancia de salto de pie y en el rendimiento de salto de rebote $(p<0,05)$. Esto sugiere, que el entrenamiento pliométrico en niños preadolescentes mejora la velocidad del sprint y la longitud del paso en la fase de velocidad máxima, relacionado con un mayor rendimiento en el salto horizontal y de rebote. 
Igualmente, Branquinho et al. (2020) examinaron el efecto de un programa de resistencia y entrenamiento pliométrico de 8 semanas, sobre diferentes habilidades motoras, velocidad de pateo y técnicas individuales, en jugadores de fútbol junior competitivos; los jugadores fueron evaluados en tres momentos: antes, durante y después del programa de entrenamiento y los resultados mostraron diferencias significativas $(p<0,05)$ en el rendimiento del sprint, salto de contra - movimiento, Yo-yo IE 2 y velocidad de golpeo; esto demuestra, que la combinación de ejercicios de fútbol y entrenamiento de potencia específico, sin tiempo de entrenamiento adicional en la temporada, optimiza el rendimiento general y específico de los futbolistas en estas edades.

De los anteriores planteamientos se deduce, que la aplicación del método de entrenamiento pliométrico puede obtener mejoras significativas sobre el desarrollo de algunas capacidades físicas y coordinativas, en niños entre los 9 y 17 años, si se aplica durante 8 semanas, con las cargas adecuadas, acordes a la edad y el desarrollo deportivo.

Entrenamiento pliométrico, agilidad y fútbol. En los estudios incluidos en esta revisión, se puede resaltar, cómo dentro del fútbol, el entrenamiento pliométrico, se ha venido fortaleciendo y tomando una nueva dirección, debido a la importancia de buscar nuevos métodos para mejorar las capacidades físicas, a la vez, que se logran avances significativos en capacidades coordinativas, en los futbolistas entre los 11 y 13 años.

Moreno (2017) afirma que "Algo interesante es el uso del entrenamiento pliométrico en deportes de equipo, como es el caso del fútbol, baloncesto, balonmano, entre otros, porque los goles, puntos o acciones decisivas vienen precedidas por aceleraciones, sprints, jugadas explosivas, saltos y disparos", acciones que requieren de fuerza, velocidad y agilidad, capacidades que pueden mejorar con este tipo de trabajo, convirtiéndolo en un factor determinante.

Para ilustrar mejor, se puede mencionar estudios, como El efecto de dos técnicas de entrenamiento pliométrico sobre la potencia muscular y la agilidad en jugadores de fútbol juvenil, realizado por Thomas et al. (2009), en el cual, su propósito fue comparar la efectividad de las modalidades de entrenamiento pliométrico de CMJ y DJ, sobre la potencia muscular y la agilidad en jugadores de fútbol. Después de la intervención, se evidenció que el entrenamiento pliométrico de DJ y CMJ puede afectar positivamente el salto vertical y el rendimiento de la agilidad en los jugadores de fútbol, sin diferencias significativas entre los modos.

Posteriormente, se encontró el estudio de Michailidis et al. (2013), denominado Entrenamiento de la pliometría en atletas de fútbol preadolescentes, que tenía como objetivo investigar, si la combinación de práctica de fútbol y entrenamiento pliométrico, mejoraría la capacidad atlética y el rendimiento específico del fútbol, en mayor medida, que solo la práctica de fútbol en jugadores pre púberes.

Con este estudio, se mostró claramente, que los niños de 10 a 11 años de edad, se pueden ver muy beneficiados por el entrenamiento pliométrico; de hecho, parece que cuando la práctica de fútbol tradicional se complementa con un protocolo de entrenamiento pliométrico supervisado y bien diseñado, puede conducir a mayores ganancias de rendimiento, que solo la práctica de fútbol y los juegos en esta edad.

De igual modo, el estudio Efecto del entrenamiento pliométrico de 16 semanas sobre acciones explosivas en jugadores de fútbol de élite de la pubertad temprana a media, por Söhnlein et al. (2014), quiso dar una recomendación sobre la duración del programa en los jugadores de fútbol de la pubertad temprana y media, con respecto a los efectos a corto plazo del entrenamiento pliométrico, para mejorar el rendimiento del sprint, la agilidad y los parámetros de salto.

Los resultados evidenciaron que el entrenamiento pliométrico parece ser el estímulo más apropiado para mejorar los parámetros de salto, la segunda fase de aceleración en el rendimiento del sprint y la agilidad general, en comparación con el entrenamiento normal de fútbol, en los deportistas de edades tempranas.

Sobre la base de las consideraciones anteriores, se puede afianzar la teoría inicial, que menciona el efecto positivo que tiene el método de entrenamiento pliométrico, en el desarrollo de la agilidad en futbolistas, entre los 11 y 13 años. Además, queda claro que debe haber un conocimiento y una estructura clara, para la implementación del mismo, en etapas formativas. 
Llegados a este punto, es importante resaltar las características que debe tener un plan de entrenamiento pliométrico (Bedoya et al. 2015).

La evidencia actual, sugiere que el entrenamiento pliométrico, se debe completar 2 días por semana, durante 8-10 semanas y durante la práctica de fútbol, con un período de descanso de 72 horas, entre días de entrenamiento pliométrico. El número inicial de contactos de pie debe ser de 50 a 60 por sesión y aumentar a no más de 80 a 120 contactos de pie por sesión, para este grupo de edad, para prevenir lesiones por uso excesivo.
Estos datos permiten tener una base para la estructuración del plan que se quiera implementar, buscando el desarrollo de diferentes habilidades en futbolistas infantiles y podría fijar un rumbo para el trabajo que desarrollan los entrenadores, de estas etapas formativas.

Finalmente, es importante resumir estos hallazgos, debido a que permiten evidenciar la relación entre entrenamiento pliométrico y agilidad (Tabla 1).

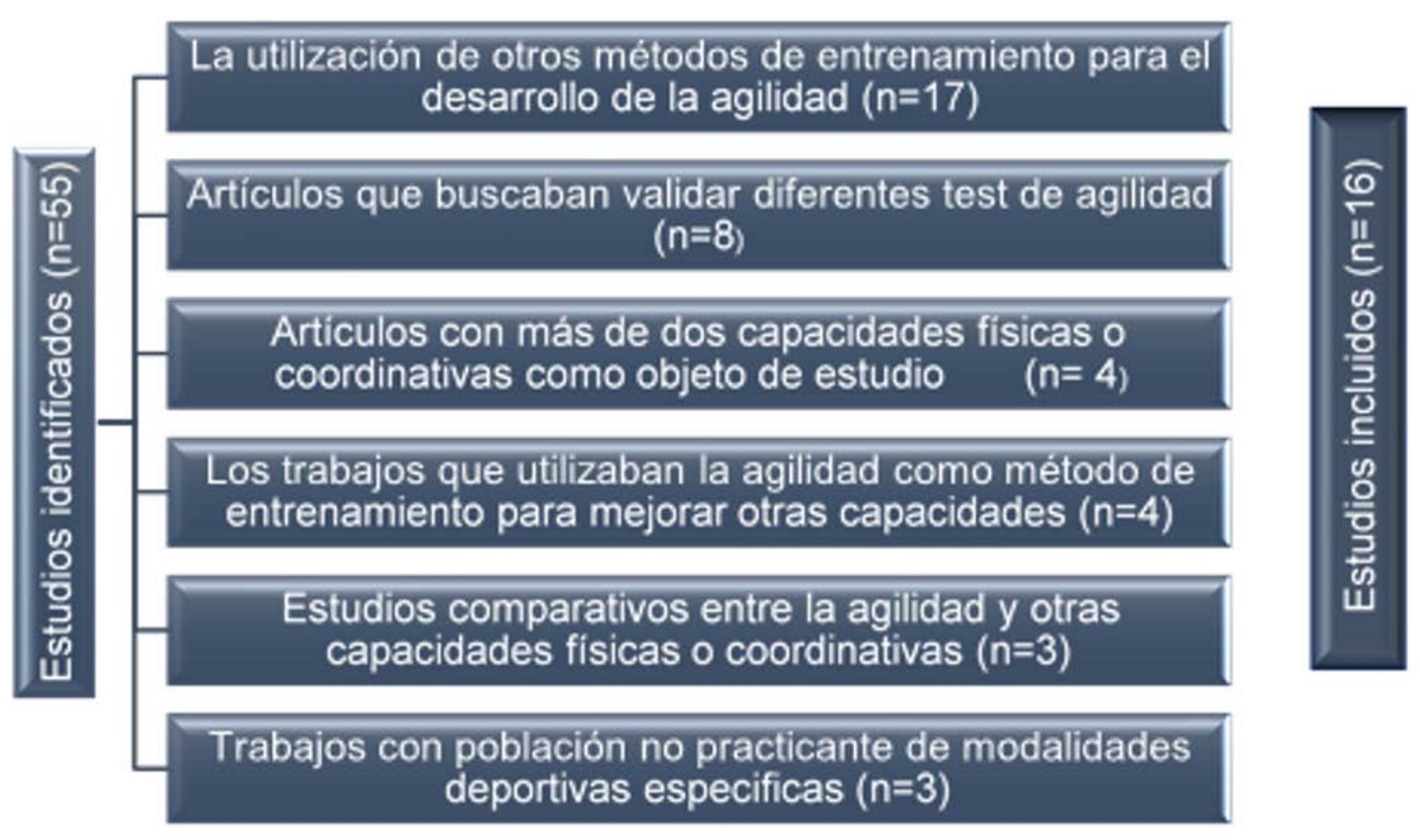

Figura 1. Procesos de selección de artículos.

El proceso de revisión bibliográfica permitió identificar las investigaciones sobre el entrenamiento pliométrico y sus efectos en la agilidad de jóvenes futbolistas; se buscaron artículos entre 2006 y 2019, en bases de datos, como PubMed, DialNet, ScienceDirect, Web of Science, Scopus, Scielo, donde se lograron identificar, inicialmente, 55 estudios; de este grupo, solo 16 cumplieron con los criterios de inclusión.

Esta literatura científica permitió analizar esta temática, desde tres aspectos importantes. El primero es la conexión que existe entre el entrenamiento pliométrico y el desarrollo de la agilidad; dentro de este punto, se encontraron artículos que demostraron los efectos positivos que tiene el método de entrenamiento pliométrico sobre la agilidad. A pesar que se han propuesto varios métodos para el desarrollo de esta capacidad, la pliometría ha tenido un valor importante, aún más, cuando simultáneamente este trabajo ayuda al desarrollo de otras capacidades físicas.

El segundo aspecto importante dentro de los resultados es la pertinencia que tiene el entrenamiento pliométrico en la formación de deportistas; en este aspecto, se logró evidenciar, cómo a través de este método, se consiguen mejoras en velocidad, fuerza explosiva, sprint y en el rendimiento del salto, en jugadores entre los 9 y 17 años.

El último aspecto importante estuvo centrado en los estudios que vincularon entrenamiento pliométrico, agilidad y fútbol; se pudo observar de qué manera este método de entrenamiento favorece el desarrollo de diferentes capacidades, como salto vertical, fuerza, aceleración, sprint, potencia y agilidad, en la formación de futbolistas, teniendo en cuenta, programas con una duración de 8 semanas, vinculados al entrenamiento regular de fútbol. 
En definitiva, con estos resultados, se podría afirmar, que el entrenamiento pliométrico puede influir, de manera positiva, en el desarrollo de la agilidad, fundamentado en los investigaciones que se identificaron en las bases de datos, en las tesis y en los libros, vinculados con programas de entrenamiento pliométrico, que se han desarrollado, a nivel nacional, regional e internacional, en el futbol masculino y que han mostrado efectos positivos en la agilidad, de acuerdo con la aplicación de programas pliométricos, durante varias semanas.

Habría que decir también, que la implementación de programas de entrenamiento pliométrico en futbolistas, entre los 11 y 13 años, que consideren el desarrollo del niño, el nivel de formación y se integren a la planificación de la temporada regular, podría brindar mayores resultados sobre la agilidad, potenciando los procesos formativos del club y brindando espacio para el trabajo de otras capacidades, en las siguientes etapas.

Por otra parte, esta revisión está centrada en identificar el desarrollo que tiene la agilidad, a través del entrenamiento pliométrico; sin embargo, sería adecuado analizar, en profundidad, los programas pliométricos aplicados dentro de cada estudio, para corroborar los resultados e ir consolidando un esquema adecuado, para el desarrollo de la agilidad, al tiempo que se propende por el bienestar y la formación adecuada de deportistas.

Finalmente, futuros estudios prácticos, a nivel nacional, enmarcados en estas tres variables, podrían contrastar o fortalecer la hipótesis de esta revisión, además, brindaría datos importantes para incluir este tipo de entrenamiento en la formación de futbolistas nacionales.

\section{CONCLUSIONES}

En cuanto a la conceptualización del término agilidad y teniendo en cuenta las diferentes nociones, a consideración del autor, se plantea la siguiente definición: La agilidad es el resultado de la combinación de fuerza, velocidad, equilibrio y coordinación, que le permiten al deportista cambiar de dirección rápidamente sin perder el balance y el control del cuerpo, respondiendo a diferentes estímulos. Dicho concepto recoge los aspectos comunes planteados por los diferentes autores y rescata algunos factores importantes, que resaltan la importancia de esta capacidad coordinativa, dentro del entrenamiento deportivo.

Con relación al método de entrenamiento pliométrico, se puede afirmar, que favorece el desarrollo de capacidades coordinativas complejas, como la agilidad, al mismo tiempo, que permite fortalecer algunas capacidades físicas en los niños futbolistas, entre los 11 y 13 años, lo que lo convierte en un método destacado para la formación de deportistas.

Asimismo, los planes de entrenamiento se deben estructurar, con una duración de 8 a 10 semanas, que se sumen al entrenamiento regular de futbol y que sean aplicados 2 veces por semana, considerando un descanso mínimo de setenta y dos horas entre cada sesión, ya que, bajo estas directrices, es donde se pueden evidenciar los resultados más destacados.

Finalmente, se puede inferir, que el trabajo de agilidad requiere un espacio diferenciado en la planificación del entrenamiento de futbolistas, con edades entre los 11 y 13 años, donde se puedan desarrollar todos los aspectos importantes de esta capacidad coordinativa compleja, a través del entrenamiento pliométrico, logrando un mejor desempeño individual, que conlleve a fortalecer el trabajo grupal.

\section{REFERENCIAS}

1. BEDOYA, A.; MILTENBERGER, M.; LÓPEZ, R. 2015. Plyometric training effects on athletic performance in youth soccer athletes: a systematic review. Journal of strength and conditioning research. 29(8):2351-2360. https://doi.org/10.1519/ jsc.0000000000000877

2. BRANQUINHO, L.; FERRAZ, R.; DUARTE MENDES, P.; PETRICIA, J.;SERRANO, J.; MARQUES, M.C. 2020. The Effect of an In-Season 8-Week Plyometric Training Programme Followed by a Detraining Period on Explosive Skills in Competitive Junior Soccer Players. Montenegrin Journal of Sports Science \& Medicine. 9(1):33-40. https://doi.org/10.26773/mjssm.200305

3. CAPPA, D. 2000. Entrenamiento de la potencia muscular. Mendoza, Argentina: Versión digital por el Grupo sobre entrenamiento. 152p. 
4. CEBAllos, J.; ZARAZA, D. 2012. Ejercicios pliométricos para incrementar la fuerza explosiva en el tren inferior de los futbolistas juveniles de la Selección Boyacá, Colombia. Revista Científica UNINCCA. 17(1):23-52.

5. CELIS, J.; SABOGAL, E. 2016. Pliometría en jóvenes tenistas. Revista de investigación: cuerpo, cultura y movimiento. 7(1):15-30.

6. CHU, D. 2006. Ejercicios pliométricos. Barcelona, España: Paidotribo. 182p.

7. COSTELLO, F.; KREIS, E. 1993. Sports agility. Taylor Sports (Nashville, Tennessee).

8. DRAPER, J.; LANCASTER, M. 1985. The 505 Test: A test for agility in the horizontal plane. Australian Journal for Science and Medicine in Sport. 17(1):15-18.

9. FISCHETTI, F.; CATALDI, S.; GRECO, G. 2019. Lowerlimb plyometric training improves vertical jump and agility abilities in adult female soccer players. Journal of Physical Education and Sport. 19(2):1254-1261. https://doi. org/10.7752/jpes.2019.02182

10. LETONA, I.; CARVALHO, H.; LEKUE, J.; BADIOLA, A.; FIGUEIREDO, A.; GIL, S. 2015. Applicability of an agility test in young players in the soccer field. Revista Brasileira de Medicina do Esporte (RBME). 21(2):133-138. https:// doi.org/10.1590/1517-869220152102144406

11. MAKHLOUF, I.; CHAOUACHI, A.; CHAUACHI, M.; BEN OTHMAN, A.; GRANACHER, U.; BEHM, D. 2018. Combination of Agility and Plyometric Training Provides Similar Training Benefits as Combined Balance and Plyometric Training in Young Soccer Players. Frontiers in Physiology. 9:1611. https://doi.org/10.3389/fphys.2018.01611

12. MEYLAN, C.; MALATESTA, D. 2009. Effects of inseason plyometric training within soccer practice on explosive actions of young players. The Journal of Strength and Conditioning Research. 23(9):2605-2613. https://doi. org/10.1519/jsc.0b013e3181b1f330
13. MICHAILIDIS, Y.; FATOUROS, I.; PRIMPA, E.; MICHAILIDIS, C.; AVLONITI, A.; CHATZINIKOLAOU, A.; BARBERO-ÁLVAREZ, J.C.; TSOUKAS, D.;DOUROUDOS, I.;DRAGANIDIS,D.; LEONTSINI, D.; MARGONIS, K.; BERBERIDOU, F.; KAMBAS, A. 2013. Plyometrics' trainability in preadolescent soccer athletes. players. The journal of strength and conditioning research. 27(1):38-49. https://doi.org/10.1519/ jsc.0b013e3182541ec6

14. MILLER, M.; HERNIMAN, J.; RICARD, M.; CHEATHAM, M.; MICHAEL, T. 2006. Efectos de un Programa de Entrenamiento Pliométrico de Seis Semanas sobre la Agilidad. Journal of Sports Science and Medicine 5(1):459-465.

15. MORENO, A. 2017. La pliometría como entrenamiento de las variables condicionales del rendimiento en futbolistas adolescentes. Moleqla revista de ciencias de la universidad pablo de olavide. 26(1):20-24.

16. MORI, I.; BHAMONDE, J.; MÉNDEZ, D. 1995. Validación de un test de agilidad, adaptado a las características anatómico-fisiológicas y posibilidades motrices del niño en primaria, apto para la valoración global de la capacidad motriz del alumno. European journal of human movement. 15(1):1-7.

17. PÉREZ, J.; MARTÍN, J.; VIVAS, J.; ALCARAZ, P. 2015. Entrenamiento de agilidad en futbolistas: una revisión sistemática. CCD. 12(1):127-134. http://dx.doi.org/10.12800/ccd.v12i35.884

18. RIVAS, M.; SÁNCHEZ, E. 2013. Fútbol, entrenamiento actual de la condición física del futbolista. MHSalud. 10(2):1-131.

19. ROOPCHAND-MARTIN, S.; LUE-CHIN, P. 2010. Plyometric Training Improves Power and Agility in Jamaica's National Netball Team. West Indian Medical Journal. 59(2):182-186.

20. ŞEKER, M.; SOSLU, R.; ÖZER, Ö. 2019. The Effect of Pliometric Training on Some Physical and Physiological Parameters. Sportive Journal. 2(2):1-9. 
21. SHEPPARD, J.; YOUNG, W. 2006. Agility literature review: Classifications, training and testing. Journal of sporte sciences. https:// doi.org/10.1080/02640410500457109

22. SÖHNLEIN, Q.; MÜLLER, E.; STÖGGL, T.L. 2014. The effect of 16-week plyometric training on explosive actions in early to midpuberty elite soccer playes. The journal of strength and conditioning research. 28(8):2105-2114. https://doi.org/10.1519/ jsc.0000000000000387

23. THOMAS, K.; FRENCH, D.; HAYES, P. 2009. The effect of two plyometric training techniques on muscular power and agility in youth soccer players. The journal of strength and conditioning research. 23(1):332-335. https:// doi.org/10.1519/jsc.0b013e318183a01a
24. TOTTORI, N.; FUJITA, S. 2019. Effects of Plyometric Training on Sprint Running Performance in Boys Aged9-12 Years. MDPIJournalsports.7(219):111. https://doi.org/10.3390/sports7100219

25. VERKHOSHANSKY, Y. 1999. Todo sobre el entrenamiento pliométrico. Barcelona, España: Editorial Paidotribo. 191p. 